\title{
Early solid tumor diagnosis through next-generation sequencing of cell-free DNA
}

\author{
Demosthenes E Ziogas ${ }^{1,2}$, loannis D Kyrochristos ${ }^{1,3}$, Efstathios G Lykoudis ${ }^{4}$ \& Dimitrios H \\ Roukos*,1,3,5 \\ ${ }^{1}$ Centre for Biosystems \& Genome Network Medicine, Ioannina University, loannina, Greece \\ ${ }^{2}$ Department of Surgery, G Hatzikosta General Hospital, Ioannina, Greece \\ ${ }^{3}$ Department of Surgery, University Hospital of Ioannina, Ioannina, Greece \\ ${ }^{4}$ Department of Plastic Surgery, University Hospital of loannina, loannina, Greece \\ ${ }^{5}$ Department of Systems Biology, Biomedical Research Foundation of the Academy of Athens (BRFAA), Athens, Greece \\ *Author for correspondence: Tel.: + 302651005 572; droukos@uoi.gr
'The most rational perspective is currently the integration of liquid biopsies by cfDNA-NGS into appropriately designed, large-scale studies and clinical trials."

First draft submitted: 8 August 2018; Accepted for publication: 21 August 2018; Published online: 31 October 2018

Keywords: cell-free DNA • early diagnosis • liquid biopsy • next-generation sequencing

Despite long-time conventional research, efforts focused on early detection of asymptomatic tumors remain unsuccessful. Over the past few years, next-generation sequencing (NGS) of circulating cell-free DNA (cfDNA) has provided most promising results on early diagnosis and, potentially, screening [1-3]. However, many challenges remain unresolved, especially in the identification of patients with stage I cancer.

The validity of NGS in human genome analysis in health $[4,5]$ and disease, with cancer as a prime example [6-9], the integration of cfDNA next-generation sequencing (cfDNA-NGS) for the first time in prenatal diagnosis [5] and the minimal clinical implications of circulating tumor cell research justify the major research focus of emerging studies in developing blood-based tests for noninvasive early detection through cfDNA-NGS [1-3].

Although mammographic screening for breast, colonoscopy for colorectal and gastroscopy for gastric cancer in high-incidence countries, such as Japan, have substantially increased the rates of early-stage detection, progress is quite slow for most other cancer types [10,11]. Considering that early disease diagnosis is of paramount importance for long-term survival or even cure, by simultaneously improving quality of life and reducing costs for public health, breakthrough research is rationally directed towards the development of blood tests with high sensitivity and specificity [12]. We summarize the advances and hurdles of cfDNA genome analysis to increase rates of resectability, including stage I cancer, and concurrently reduce the incidence of locally advanced or metastatic tumors.

\section{Modern standards \& challenges}

During the past decades, overall survival rates have been substantially improved through diagnosis in earlier rather than later tumor stages. Nevertheless, this success is limited only to a few cancer types, including breast, primary liver and gastric cancer in Japan [10]. Increased rates of stage I diagnosis have shifted treatment from aggressive multimodal therapy, including surgery, chemotherapy, radiotherapy and targeted therapy to less invasive strategies, such as wide local excision for breast cancer and endoscopic mucosal resection for early gastric cancer (T1a). Therefore, not only long-term oncological outcomes, but also quality of life, have dramatically improved.

However, a significant proportion of patients continue to be diagnosed in advanced or metastatic settings and, additionally, highly aggressive tumors, including pancreatic, esophageal and bile duct cancers, are associated with low rates of stage I/II detection. Despite initial enthusiasm to the putative diagnostic utility of circulating tumor cells in the past decades, results remain largely unsuccessful. These unmet needs could potentially be overcome through understanding tumorigenesis to develop new molecular methods for clinical early disease detection. The most rational perspective is currently the integration of liquid biopsies by cfDNA-NGS into appropriately designed, large-scale studies and clinical trials. 


\section{Early noninvasive diagnosis: from small studies to large-scale clinical trials}

Recently, significant research resources have been assigned in the investigation of liquid biopsies through cfDNANGS as markers of early cancer diagnosis with major potential clinical implications. Early small-scale efforts on diverse tumor types and tumor stages have suggested the potential diagnostic utility of cfDNA-NGS [12-14], reporting acceptable rates for noninvasive diagnosis. In specific, Shutz et al. [13] investigated copy number variation as a diagnostic marker, analyzing 204 blood samples from prostate cancer patients with the SOLID-P2 NGS platform. A mathematical model was constructed based on molecular characteristics that achieved cancer detection with a reported accuracy of $83 \%$. However, most studies have correlated cfDNA levels and, thus, cfDNA-NGS feasibility with tumor burden $[15,16]$, highlighting the technical hurdles in the detection of cancer at early versus advanced or metastatic stages. Therefore, despite highly promising preliminary results and seemingly good performance in the detection of late-stage cancer, the utility of cfDNA as a diagnostic tool remains controversial, especially in the early stages of disease.

Consequently, considering the potential clinical, as well as financial, impact of developing a noninvasive, patientfriendly blood test for cancer diagnosis, latest research endeavors are concentrated on conducting large-scale studies to clarify whether cfDNA represents a robust and effective diagnostic tool. Assessing this research opportunity, Cohen et al. designed a cost-effective ( $<500$ dollar cost) multi-analyte blood test for the detection of eight common cancer types, aiming for early-stage diagnosis and reducing cancer-related deaths [1]. This prototype assay, termed CancerSEEK, utilizes mutation analysis of 16 genes and identification of eight protein biomarkers in conjunction, reportedly significantly enhancing diagnostic accuracy, compared with isolated genetic testing and allowing for tumor localization for a variety of resectable cancers. This comes in direct contrast to the vast majority of previous smaller studies, which evaluated liquid biopsies in advanced and metastatic settings [12].

In total, 1005 patients with eight different, nonmetastatic, stage I to III cancer types and 812 healthy controls were included in the analysis. The most common disease stage was tumor-node-metastasis (TNM) stage II (49\%), while stages I and III accounted for 20 and 31\% of cases, respectively. Overall, median sensitivity was reported at $70 \%$, with the lowest rate observed for breast cancer at $33 \%$ and the highest for liver and ovarian cancers at approximately 98\%. Rates ranged between 69 and 98\% for five tumor types, namely esophageal, gastric, pancreatic, liver and ovarian cancers, without screening test available. However, sensitivity rates for stage I tumors were only $43 \%$. Specificity was very high, measured over 99\%, with only seven false positive results out of 812 controls. In 153 patients, from whom tissue samples were available, tissue-plasma mutational concordance was $90 \%$. Moreover, tumor localization in a single organ was feasible in $63 \%$ of positive patients, mostly based on data derived from the protein markers, since driver mutations are nontissue specific. Thus, the CancerSEEK test has provided proofof-concept for noninvasive cancer diagnosis through liquid biopsies. Nevertheless, it faces two major limitations. First, most enrolled patients were diagnosed with symptomatic disease, and therefore the examined population is not representative of the screening setting, where most individuals bare earlier disease. And second, the proportions of each of the eight cancer types do not reflect the cancer's true incidence in the general population, leading to a mathematical sensitivity estimation of 55\%, when weighted for actual incidence [1].

Recently, a substudy on pancreatic cancer was also published [2]. The cohort included 221 patients with resectable pancreatic ductal adenocarcinoma and 182 controls. The majority of tumors were stage IIB (77\%). Blood testing for KRAS mutations yielded a sensitivity of only $30 \%$. These rates were more than doubled to $64 \%$ overall by combining KRAS mutation analysis with four protein biomarkers (CA 19-9, CEA, HGF, OPN). However, detection rates for stage IA and IB were 33 and 47\%, respectively, while they did not surpass 50\% even for stage IIA. This can be explained by the very low numbers of mutant plasma templates. The correlation of plasma cfDNA levels to tumor burden has indeed already been established by a multitude of smaller studies [12], suggesting that highly sensitive techniques and further technical refinements are required to develop an effective liquid biopsy-based diagnostic approach for early-stage cancer. Another interesting finding was that patients who tested positive tended to experience worse outcomes, indicating a potential prognostic utility. However, it remains controversial whether such a test could substantially improve patient survival, even following future optimization for earlier detection [2].

Within this context, the first clinical trial on the diagnostic utility of cfDNA-based liquid biopsies, titled 'The Circulating Cell-free Genome Atlas Study (CCGA)', was registered in 2016 (NCT02889978). The multicenter study has already enrolled more than 12,000 of the planned 15,000 participants. The study employs three prototype sequencing assays for the noninvasive detection of a variety of cancers, namely targeted NGS (tNGS), whole-genome sequencing (WGS) and whole-genome bisulfite sequencing (WGBS). Preliminary results make promises for up 
to $90 \%$ accuracy in the detection of ten different cancer types and widespread commercial availability in 5-10 years. The best reported results were for ovarian, pancreatic and liver cancer, with sensitivity reaching 90,80 and $80 \%$, respectively. The test showed intermediate sensitivity, ranging between 77 and $56 \%$, for other cancer types, including lymphoma, multiple myeloma, bowel, esophageal, lung, triple-negative breast cancer (TNBC), as well as head and neck cancers. However, the noninvasive test was less effective in the diagnosis of other major tumors, such as breast, gastric, uterine and early-stage low-grade prostate cancer [17].

Recently the preliminary results on breast and lung cancers were presented at the 2018 ASCO annual meeting [3,18]. More specifically, the substudy on breast cancer included blood samples from 358 patients with newly diagnosed invasive breast cancer and 452 healthy individuals with similar baseline characteristics. Symptomatic patients consisted $46 \%$ of the cohort and $82 \%$ of tumors were stage I/II. TNBC represented $65 \%$ of tumors, while hormone receptor (HR) and HER2 positive cancers stood for 17 and 15\% of the total. The best rates of sensitivity out of the three assays were achieved by WGBS, at 58\% for TNBC, 40\% for HER2-positive and 15\% for HR-positive/HER2-negative, while detection rates for asymptomatic cancers were only $10 \%$ [3]. Similarly, preliminary results on 127 patients with lung cancer versus 580 controls have been reported. Best sensitivity rates were once again achieved with WGBS and were 56\% for early-stage (stage I-IIIA) and 93\% for late-stage (stage IIIB-IV) cancers, irrespective of the histological subtype [18]. In both subanalyses tNGS and WGS consistently featured lower sensitivity. Therefore, these data suggest that cfDNA-based liquid biopsies could potentially act as diagnostic markers, even in the early stages of cancer, following validation within further large-scale clinical trials and further technical refinements to improve accuracy.

Evidently, returned results from the CancerSEEK test are consistent with data provided by the CCGA regarding the high sensitivity of noninvasive diagnosis for ovarian, liver and pancreatic cancers, whereas sensitivity has proven moderate for cancers such as colorectal and lung tumors. Future trials are required to confirm these data or provide solutions on how to increase accuracy for cancers with moderate or low sensitivity rates.

\section{Future perspective $\&$ conclusion}

Moving from small studies on patient-derived plasma samples to large-scale cfDNA-NGS analyses $[1,2]$ and clinical trials [3], progress in noninvasive diagnosis has revolutionized biomedical research on cancer management. Considering that tumor resectability is an independent positive prognostic factor for survival and that rates of early diagnosis continue to be modest, it comes indeed as no surprise that blood testing to identify patients with early-stage tumors represents a high priority.

Available data suggest that liquid biopsy-based diagnosis can be distinguished into three categories. First, tumor types such as ovarian, hepatocellular and pancreatic, are associated with high sensitivity rates. Second, other cancer types, including colorectal, esophageal, lung and TNBC could be diagnosed with moderate accuracy. Third, for some common cancer types, such as breast, gastric and prostate, recent data report disappointing results. How could these hurdles be overcome, considering sensitivity, specificity and tumor localization?

The variability in sensitivity rates among different cancer types confirms the results of the ENCODE project $[4,19]$ on cell- and organ-specificity of genome functionality and guides cancer type-specific trials. New trials are required both to establish blood testing as an early diagnostic tool. How could the moderate and low sensitivity rates be significantly improved? First, the expected completion of the comprehensive catalog of genes involved in each distinct tumor could improve sensitivity through the expansion of the integrated gene panels in analyses utilizing tNGS of cfDNA. This potential is supported by the established extensive genetic heterogeneity [20]. Second, other alternatives include the breakthrough combination of tNGS and protein biomarkers, based on the recent success of the CancerSEEK test $[1,2]$. Third, two future solutions could be presented by technical refinements in cfDNA detection and the integration of functional noncoding mutations and understanding of regulatory networks.

\section{Increasing the rates of early diagnosis}

Considering the evidence on interpatient genomic heterogeneity [7,21], besides the establishment of genetic variation [20], upgrading current cfDNA-tNGS approaches to WGS could increase diagnostic accuracy in two ways. First, tumoral WGS analyses have already established large structural genome changes, such as genomic rearrangements and copy number alterations, as crucial components of tumor heterogeneity. Assuming that such alterations can only be identified through WGS, cfDNA-WGS could dramatically increase early diagnosis. And second, beyond these structural variations, functional mutations in noncoding genomic areas also contribute to interpatient hetero- 
geneity [22]. Therefore, inclusion of the noncoding regulatory mutational landscape in the design of cfDNA-NGS studies could potentially improve early disease detection rates.

Ultimately, exploring both the structural and functional noncoding mutational landscapes and their integration into heterogeneous temporal regulatory networks [23-25] might also, in the more distant future, enhance sensitivity and tumor localization, within comprehensive analyses of cfDNA. However, it should be emphasized that the greatest challenge for early, stage I diagnosis and screening is the very small burden of circulating cfDNA escaping from the small primary tumor.

In summary, recent data on noninvasive tests integrating NGS of cell-free DNA in the circulation suggest that liquid biopsies might provide a useful diagnostic tool for patients with resectable tumors. Nevertheless, while high sensitivity rates have been achieved for some cancer types, testing results are quite disappointing for several major tumor types, such as breast cancer [17]. Moreover, blood testing for cancer detection at the early stages of tumorigenesis for potential population screening remains elusive, due to the small tumor bulk resulting in very low plasma cfDNA levels, undetectable with current methods.

Noninvasive early diagnosis has a central role in the optimization of cancer care. Based on the extensive, not only genetic, but also genomic, heterogeneity the development of a cfDNA-WGS platform for the additional identification of large genomic rearrangements, copy number aberrations and functional noncoding alterations could remarkably increase diagnostic accuracy. Furthermore, technical refinement of a breakthrough cfDNA-WGS system could also potentially enable stage I tumor detection in asymptomatic patients for screening purposes.

\section{Financial \& competing interests disclosure}

The authors have no relevant affiliations or financial involvement with any organization or entity with a financial interest in or financial conflict with the subject matter or materials discussed in the manuscript. This includes employment, consultancies, honoraria, stock ownership or options, expert testimony, grants or patents received or pending, or royalties.

No writing assistance was utilized in the production of this manuscript.

\section{References}

1. Cohen JD, Li L, Wang Y et al. Detection and localization of surgically resectable cancers with a multi-analyte blood test. Science 359(6378), 926-930 (2018).

2. Cohen JD, Javed AA, Thoburn C et al. Combined circulating tumor DNA and protein biomarker-based liquid biopsy for the earlier detection of pancreatic cancers. Proc. Natl Acad. Sci. USA 114(38), 10202-10207 (2017).

3. Liu MC, Maddala T, Aravanis A et al. Breast cancer cell-free DNA (cfDNA) profiles reflect underlying tumor biology: the Circulating Cell-Free Genome Atlas (CCGA) study. Presented at: ASCO Annual Meeting 2018, IL, USA, 1-5 June 2018.

4. An integrated encyclopedia of DNA elements in the human genome. Nature 489(7414), 57-74 (2012).

5. Shendure J, Balasubramanian S, Church GM et al. DNA sequencing at 40: past, present and future. Nature 550(7676), 345-353 (2017).

6. Swanton C, Soria JC, Bardelli A et al. Consensus on precision medicine for metastatic cancers: a report from the MAP conference. Ann. Oncol. 27(8), 1443-1448 (2016).

7. Roukos DH. Spatiotemporal diversification of intrapatient genomic clones and early drug development concepts realize the roadmap of precision cancer medicine. Drug Discov. Today 22(8), 1148-1164 (2017).

8. The Cancer Genome Atlas (2018). https://cancergenome.nih.gov/

9. International Cancer Genome Consortium (2018). http://icgc.org/

10. Siegel RL, Miller KD, Jemal A. Cancer statistics, 2018. CA Cancer J. Clin. 68(1), 7-30 (2018).

11. National Comprehensive Cancer Network, Inc. (2018). www.nccn.org/

12. Ziogas DE, Kyrochristos ID, Roukos DH. Discovering novel valid biomarkers and drugs in patient-centric genomic trials: the new epoch of precision surgical oncology. Drug Discov. Today 2(18) 30250-30252 2018).

13. Schutz E, Akbari MR, Beck J et al. Chromosomal instability in cell-free DNA is a serum biomarker for prostate cancer. Clin. Chem. 61(1), 239-248 (2015).

14. Holmila R, Sklias A, Muller DC et al. Targeted deep sequencing of plasma circulating cell-free DNA reveals Vimentin and Fibulin 1 as potential epigenetic biomarkers for hepatocellular carcinoma. PLoS ONE 12(3), e0174265 (2017).

15. Ohira T, Sakai K, Matsubayashi J et al. Tumor volume determines the feasibility of cell-free DNA sequencing for mutation detection in non-small cell lung cancer. Cancer Sci. 107(11), 1660-1666 (2016).

16. Vandekerkhove G, Todenhofer T, Annala M et al. Circulating tumor DNA reveals clinically actionable somatic genome of metastatic bladder cancer. Clin. Cancer Res. 23(21), 6487-6497 (2017). 
17. Mail Online (2018). www. dailymail.co.uk/health/article-5791793/Blood-test-detect-TEN-types-cancer-YEARS-ill.html

18. Oxnard GR, Maddala T, Hubbell E et al. Genome-wide sequencing for early stage lung cancer detection from plasma cell-free DNA (cfDNA): the Circulating Cancer Genome Atlas (CCGA) study. Presented at: ASCO Annual Meeting 2018, IL, USA, 1-5 June 2018.

19. Gerstein MB, Kundaje A, Hariharan M et al. Architecture of the human regulatory network derived from ENCODE data. Nature 489 (7414), 91-100 (2012)

20. Lawrence MS, Stojanov P, Mermel CH et al. Discovery and saturation analysis of cancer genes across 21 tumour types. Nature 505 (7484), 495-501 (2014).

21. Tirosh I, Izar B, Prakadan SM et al. Dissecting the multicellular ecosystem of metastatic melanoma by single-cell RNA-seq. Science 352(6282), 189-196 (2016).

22. Rheinbay E, Parasuraman P, Grimsby J et al. Recurrent and functional regulatory mutations in breast cancer. Nature 547 (7661), 55-60 (2017).

23. Roukos DH. Crossroad between linear and nonlinear transcription concepts in the discovery of next-generation sequencing systems-based anticancer therapies. Drug Discov. Today 21(4), 663-673 (2016).

24. Li A, Cornelius SP, Liu YY, Wang L, Barabasi AL. The fundamental advantages of temporal networks. Science 358(6366), 1042-1046 (2017).

25. Mod EC, Roy S, Ernst J et al. Identification of functional elements and regulatory circuits by Drosophila modENCODE. Science 330(6012), 1787-1797 (2010). 
